\title{
Normal forms and entanglement measures for multipartite quantum states
}

\author{
Frank Verstraete, Jeroen Dehaene and Bart De Moor \\ Katholieke Universiteit Leuven, Department of Electrical Engineering, Research Group SISTA \\ Kasteelpark Arenberg 10, B-3001 Leuven, Belgium
}

(Dated: August 6, 2001)

\begin{abstract}
A general mathematical framework is presented to describe local equivalence classes of multipartite quantum states under the action of local unitary and local filtering operations. This yields multipartite generalizations of the singular value decomposition. The analysis naturally leads to the introduction of entanglement measures quantifying the multipartite entanglement (as generalizations of the concurrence and the 3-tangle), and the optimal local filtering operations maximizing these entanglement monotones are obtained. Moreover a natural extension of the definition of GHZ-states to e.g. $2 \times 2 \times N$ systems is obtained.
\end{abstract}

One of the major challenges in the field of quantum information theory is to get a deep understanding of how local operations assisted by classical communication (LOCC) performed on a multipartite quantum system can affect the entanglement between the spatially separated systems. In this paper we investigate this problem in the case that only operations on one copy of the system are allowed. This is different from the general setup of entanglement distillation, where global operations on a large (infinite) number of copies are performed to concentrate the entanglement in a few copies. The main motivation of this work was to characterize the optimal filtering operations (SLOCC) to be performed on one copy of a multipartite system such that, with a non zero chance, a state with maximal possible entanglement is obtained. In other words, we want to design the optimal filtering operations for a given state, such that with a certain chance we prepare the optimal attainable one. Of course this leads to the introduction of local equivalence classes.

In the case of a pure state of two qubits, this optimal filtering procedure is commonly known as the Procrustean method [1]. Following the work of Gisin [2], Horodecki [3],Linden et al. [4] and Kent et al. [5, 6], the optimal filtering procedure for mixed states of two qubits was recently derived in [7]. In this paper we extend these ideas to pure and mixed multipartite systems of qudits of arbitrary dimension.

The optimal filtering operations in [7] were derived by proving the existence of a decomposition of a mixed state of two qubits as a unique Bell diagonal state multiplied left and right by a tensor product representing local operations. A Bell diagonal state is special in the sense that one party alone cannot acquire any information at all about the state: its local density operator is equal to the identity. This can readily be generalized to multipartite systems of arbitrary dimensions, and the existence of local operations transforming a generic state to a unique state with all local density operators equal to the identity will be proved. In the case of pure states, this decomposition leads to a transparent method of deriving essentially different states such as GHZ- and W-states [8].
We then proceed to show that all quantities exhibiting some kind of invariance under the considered SLOCC operations are entanglement monotones [9]. It is shown that the concurrence and the 3-tangle, introduced by Wootters et al. 10, 11], belong to this class of entanglement measures. Therefore a natural generalization of these measures is obtained to systems of arbitrary dimensions and an arbitrary number of parties.

A subsequent part of the paper is concerned with finding the optimal filtering operations for a given multipartite state. It is shown that the SLOCC operations bringing a state into its unique normal form maximize all the introduced entanglement monotones. This was expected in the light of the work by Nielsen about majorization [12]: the notion of local disorder is intimately connected to the existence of entanglement.

Finally, the appendix presents some results on the characterization of local unitary equivalence classes, yielding a natural and constructive but non-unique generalization of the singular value decomposition to the multilinear setting.

\section{Normal forms under SLOCC operations}

Let us first consider the case of pure states. The main goal is to study equivalence classes under general local transformations of the kind $\left|\psi^{\prime}\right\rangle=A_{1} \otimes \cdots \otimes A_{n}|\psi\rangle$ with $\left\{A_{i}\right\}$ arbitrary matrices. These kind of transformations are called SLOCC transformations [8] (from stochastic local operations assisted by classical communication), and are also called local filtering operations. It will turn out very useful to restrict ourselves to SLOCC transformations where all $\left\{A_{i}\right\}$ are full rank (remark that entanglement is lost whenever a $A_{i}$ is not full rank). For convenience, we will consider all $\left\{A_{i}\right\}$ to belong to $S L(n, \mathcal{C})$, the group of square complex matrices having determinant equal to 1 , and consider unnormalized states.

Let us formulate the following central theorem:

Theorem 1 Consider an $N_{1} \times N_{2} \times \cdots N_{p}$ pure multipartite state (or tensor). Then this state (tensor) can con- 
structively be transformed into a normal form by determinant 1 SLOCC operations. The local density operators of the normal form are all proportional to the identity, and the normal form is unique up to local unitary transformations. Moreover, the state connected to the original one by determinant 1 SLOCC operations with the minimal possible norm (i.e. trace of the unnormalized density operator) is in normal form.

Proof: We will give a constructive proof of this theorem that can directly be translated into matlab code. The idea is that the local determinant 1 operators $A_{i}$ bringing $\psi$ into its normal form can be iteratively determined by a procedure where at each step the trace of $|\psi\rangle\langle\psi|=\rho$ is minimized by a local filtering operation of one party. Consider therefore the partial trace $\rho_{1}=\operatorname{Tr}_{2 \cdots p}(\rho)$. If $\rho_{1}$ is full rank, there exists an operator $X$ with determinant 1 such that $\rho_{1}^{\prime}=X \rho_{1} X^{\dagger} \sim I_{N_{1}}$. Indeed, $X=\left|\operatorname{det}\left(\rho_{1}\right)\right|^{1 / 2 N_{1}}\left(\sqrt{\rho_{1}}\right)^{-1}$ does the job 19], and we have $\rho_{1}^{\prime}=\operatorname{det}\left(\rho_{1}\right)^{1 / N_{1}} I_{N_{1}}$. We also have the relation:

$$
\operatorname{Tr}\left(\rho^{\prime}\right)=N_{1} \operatorname{det}\left(\rho_{1}\right)^{1 / N_{1}} \leq \operatorname{Tr}\left(\rho_{1}\right),
$$

where $\rho^{\prime}=(X \otimes I \cdots \otimes I)|\psi\rangle\langle\psi|(X \otimes I \cdots \otimes I)^{\dagger}$. This inequality follows from the fact that the geometric mean is always smaller than the arithmetic mean, with equality iff $\rho_{1}$ is proportional to the identity. Therefore the trace of $\rho$ decreases after this operation. We can now repeat this procedure with the other parties, and then repeat everything iteratively over and over again. After each iteration, the trace of $\rho$ will decrease unless all partial traces are equal to the identity. Because the trace of a positive definite operator is bounded from below, we know that the decrements become arbitrarily small and following equation (11) this implies that all partial traces converge to operators arbitrarily close to the identity.

We still have to consider the case where we encounter a $\rho_{i}$ that is not full rank. Then there exists a series of $X$ whose norm tends to infinity but has determinant 1 such that $X \rho_{i} X^{\dagger}=0$, leading to a normal form identical to zero, clearly the positive operator with minimal possible trace. This ends the proof of the existence of the normal form.

Consider now the a state that is normal form; then due to the construction of the proof, the trace can always be decreased by determinant 1 SLOCC operations, unless the state is in normal form.

As pointed out by Briand, Luque and Thibon [13], the normal form is unique up to local unitaries: the KempfNess criterion proves the uniqueness in the case of a closed orbit, and there is always a unique closed orbit in the closure of an arbitrary orbit [14]. This ends the proof.

Let us now return to the general theorem 1] This theorem is very fundamental in that it states that each pure multipartite state can be transformed into a unique state with the property that all local density operators are proportional to the identity. States in normal form are clearly expected to be maximally entangled states. As we will argue later, the normal form is the state with the maximal amount of entanglement that can be created locally and probabilistically from the original state.

Let us next prove that the normal form is continuous with respect to perturbations of the entries of the original density matrix $\rho$. First of all note that the nonuniqueness due to the local unitaries can be circumvented by imposing all $A_{i}$ to be hermitian. The following lemma shows that the normal form is robust against perturbations or noise:

Lemma 1 If the SLOCC operations bringing the state into the normal form introduced in theorem 1 are chosen to be hermitian, and if they turn out to be finite, then the normal form is continuous with respect to the entries of the state.

Proof: Let us consider $\rho=\left(A_{1} \otimes \cdots \otimes A_{p}\right) \sigma\left(A_{1} \otimes \cdots \otimes\right.$ $\left.A_{p}\right)^{\dagger}$ and a perturbation $\dot{\rho}$ resulting in $\left\{\dot{A}_{i}\right\}$ and $\dot{\sigma}$. The following formula is readily verified:

$$
\begin{aligned}
& \left(A_{1} \otimes \cdots \otimes A_{p}\right)^{-1} \dot{\rho}\left(A_{1} \otimes \cdots \otimes A_{p}\right)^{-\dagger}= \\
& \dot{\sigma}+\sum_{i=1}^{p}\left(\left(I \otimes \cdots A_{i}^{-1} \dot{A}_{i} \cdots \otimes I\right) \sigma+\text { h.c. }\right) .
\end{aligned}
$$

As all $\left\{A_{i}\right\}$ are hermitian and have determinant 1 , all $A_{i}^{-1} \dot{A}_{i}$ are skew-hermitian and the second term lives in another subspace $S_{2}$ than the first term $\dot{\sigma}$ who lives in subspace $S_{1} . \dot{\sigma}$ can therefore be obtained by projecting the left hand side parallel to $S_{2}$ onto $S_{1}$. As $\dot{\rho}$ is finite and all $\left\{A_{i}\right\}$ have determinant one and are finite, this projection is of course also finite. This proofs that $\dot{\sigma}$ is of the same order of magnitude as $\dot{\rho}$, which ends the proof.

Note that we have also proven continuity with respect to mixing.

Let us now discuss some peculiarities. The fact that the algorithm can converge to zero despite the fact that all $A_{i}$ have determinant equal to 1 is a consequence of the fact that $S L(n, \mathcal{C})$ is not compact: there exist states that can only be brought into their respective normal form by infinite transformations, although the class of states with this property is clearly of measure zero. As an example consider the $W$-state [8] $|\psi\rangle=|001\rangle+|010\rangle+|100\rangle$. The following identity is easily checked:

$$
\lim _{t \rightarrow \infty}\left(\begin{array}{cc}
1 / t & 0 \\
0 & t
\end{array}\right)^{\otimes 3}|W\rangle=0
$$

The normal form corresponding to the $W$-state is therefore equal to zero, clearly the state with the minimal possible trace. This is interesting, as it will be shown that a normal form is zero iff a whole class of entanglement 
monotones is equal to zero. Therefore the states with normal form equal to zero are fundamentally different from those with finite normal form, and this leads to the generalization of the $W$-class to arbitrary dimensions.

It thus happens that some states have normal form equal to 0 . This also happens if the state does not have full support on the Hilbert space in that one partial trace $\rho_{i}$ is rank deficient. Note that states which do not have full support on the Hilbert space, such as pure states from which one party is fully separable, all have normal form equal to zero. It will indeed turn out that the amount of multipartite entanglement present in a state can be quantified by the trace of the obtained normal form, which is clearly zero in the case of separable states. On the other hand, the only normalized states that are already in normal form are precisely the maximally entangled states: in the case of three qubits for example, the only state with the property that all its local density operators are proportional to the identity is the GHZ-state.

As a last remark, we give an example of a state that is brought into a non-zero normal form by SLOCC operators that are unbounded:

$$
|\psi\rangle \simeq a(|0000\rangle+|1111\rangle)+|01\rangle(|10\rangle+|01\rangle)
$$

The normal form is just given by the GHZ-state $(|0000\rangle+$ $|1111\rangle)$, but as can be derived from the results presented in [15], infinite SLOCC transformations are needed to reach this.

\section{Entanglement monotones}

Until now we contented ourselves to characterize the orbits generated by local unitary or SLOCC operations, but we have not tried to quantify the entanglement present in a state. The SLOCC normal form introduced in the previous section however gives us a strong hint of how to do this. Note that all separable states have a normal form equal to zero, and that the known maximally entangled states such as Bell-states and GHZ-states are the only ones of their class that are in normal form.

This suggests a very general way of constructing entanglement monotones:

Theorem 2 Consider a linearly homogeneous positive function of a pure (unnormalized) state $M(\rho=|\psi\rangle\langle\psi|)$ that remains invariant under determinant 1 SLOCC operations. Then $M(|\psi\rangle\langle\psi|)$ is an entanglement monotone.

Proof: A quantity $M(\rho)$ is an entanglement monotone iff its expected value does not increase under the action of every local operation. It is therefore sufficient to show that for every local $A_{1} \leq I_{N_{1}}, \bar{A}_{1}=\sqrt{I_{N_{1}}-A_{1}^{\dagger} A_{1}}$, it holds that

$$
M(\rho) \geq \operatorname{Tr}\left(\left(A_{1} \otimes I\right) \rho\left(A_{1} \otimes I\right)^{\dagger}\right) .
$$

$$
\begin{array}{r}
M\left(\frac{\left(A_{1} \otimes I\right) \rho\left(A_{1} \otimes I\right)^{\dagger}}{\operatorname{Tr}\left(\left(A_{1} \otimes I\right) \rho\left(A_{1} \otimes I\right)^{\dagger}\right)}\right) \\
+\operatorname{Tr}\left(\left(\overline{A_{1}} \otimes I\right) \rho\left(\overline{A_{1}} \otimes I\right)^{\dagger}\right) . \\
M\left(\frac{\left(\overline{A_{1}} \otimes I\right) \rho\left(\overline{A_{1}} \otimes I\right)^{\dagger}}{\operatorname{Tr}\left(\left(\overline{A_{1}} \otimes I\right) \rho\left(\overline{A_{1}} \otimes I\right)^{\dagger}\right)}\right)
\end{array}
$$

If $A_{1}$ is full rank, it can be transformed to a determinant 1 matrix by dividing it by $\operatorname{det}\left(A_{1}\right)^{1 / N_{1}}$. Due to the homogeneity of $M(\alpha \rho)=\alpha M(\rho)$ the previous inequality is equivalent to

$$
M(\rho) \geq\left(\left|\operatorname{det}\left(A_{1}\right)\right|^{2 / N_{1}}+\left|\operatorname{det}\left(\overline{A_{1}}\right)\right|^{2 / N_{1}}\right) M(\rho) .
$$

As the arithmetic mean always exceeds the geometric mean, this inequality is always satisfied. This argument can be easily completed to the cases where $A_{i}$ is not full rank due to continuity. The same argument can then be repeated for the other $A_{i}$, which ends the proof.

Entanglement monotones of the above class can readily be constructed using the completely antisymmetric tensor $\epsilon_{i_{1} \cdots i_{N}}$.

Indeed, it holds that $\sum A_{i_{1} j_{1}} A_{i_{2} j_{2}} \cdots A_{i_{N} j_{N}} \epsilon_{j_{1} \cdots j_{N}}=$ $\operatorname{det}(A) \epsilon_{i_{1} \cdots i_{N}}$, and as $\operatorname{det}(A)=1$ this leads to invariant quantities under determinant 1 SLOCC operations. These quantities seem to be related to hyperdeterminants 16? ], and those latter seem to be a subclass of the quantities considered here.

Consider for example the case of two qubits. The quantity

$$
\left|\sum_{i_{1} j_{1} i_{2} j_{2}} \psi_{i_{1} j_{1}} \psi_{i_{2} j_{2}} \epsilon_{i_{1} i_{2}} \epsilon_{j_{1} j_{2}}\right|
$$

is clearly of the considered class, and it happens to be the celebrated concurrence entanglement measure [1]. In the case of three qubits, the simplest non-trivial homogeneous quantity invariant under determinant 1 SLOCC operations is given by

$\left|\psi_{i_{1} j_{1} k_{1}} \psi_{i_{2} j_{2} k_{2}} \psi_{i_{3} j_{3} k_{3}} \psi_{i_{4} j_{4} k_{4}} \epsilon_{i_{1} i_{2}} \epsilon_{i_{3} i_{4}} \epsilon_{j_{1} j_{2}} \epsilon_{j_{3} j_{4}} \epsilon_{k_{1} k_{3}} \epsilon_{k_{2} k_{4}}\right|^{1 / 2}$

(Note that we use the Einstein summation convention.) This happens to the square root of the 3-tangle introduced by Wootters et al. 10], which quantifies the true tripartite entanglement.

More generally, as the considered entanglement monotones are invariant under the determinant 1 SLOCC operations, the number of independent entanglement monotones is equal to the degrees of freedom of the normal form obtained in the case of a pure state minus the degrees of freedom induced by the local unitary operations. Indeed, this is the amount of invariants of the whole class of states connected by SLOCC operations. It is then easily proven that a normal form is equal to zero if and only if all the considered entanglement monotones are equal to zero: the entanglement monotones are homogeneous 
functions of the normal form, and if the normal form is not equal to zero there always exists an SLOCC invariant quantity that is different from zero.

In the case of 4 qubits for example, parameter counting leads to $\left(2 \cdot 2^{4}-2\right)-4 \cdot 6=6$ (a state has 32 degrees of freedom -2 to an irrelevant phase and the $4 S L(2, \mathcal{C})$ matrices have each 6 degrees of freedom) independent entanglement monotones. The simplest monotone is given by

$$
\left|\psi_{i_{1} j_{1} k_{1} l_{1}} \psi_{i_{2} j_{2} k_{2} l_{2}} \epsilon_{i_{1} i_{2}} \epsilon_{j_{1} j_{2}} \epsilon_{k_{1} k_{2}} \epsilon_{l_{1} l_{2}}\right|
$$

and the other 5 entanglement monotones can be obtained by including more factors; an example is

$$
\begin{aligned}
& \sqrt{2} \mid \psi_{i_{1} j_{1} k_{1} l_{1}} \psi_{i_{2} j_{2} k_{2} l_{2}} \psi_{i_{3} j_{3} k_{3} l_{3}} \psi_{i_{4} j_{4} k_{4} l_{4}} . \\
& \left.\quad \epsilon_{i_{1} i_{2}} \epsilon_{i_{3} i_{4}} \epsilon_{l_{1} l_{2}} \epsilon_{l_{3} l_{4}} \epsilon_{j_{1} j_{3}} \epsilon_{j_{2} j_{4}} \epsilon_{k_{1} k_{3}} \epsilon_{k_{2} k_{4}}\right|^{1 / 2}
\end{aligned}
$$

These are clearly generalizations of the concurrence and the 3-tangle to four parties. Note however that the situation here is more complicated due to the existence of multiple independent entanglement monotones. Note also that there exist biseparable states that can be brought into a non-zero normal form by determinant 1 SLOCC operations. Consider for example the tensor product of two Bell states; all local density operators are proportional to the identity, the value of the entanglement monotones (3) and (4) is respectively given by 1 and $1 / \sqrt{2}$ (as opposed to 1 and 1 for the GHZ-state $(|0000\rangle+|1111\rangle) / \sqrt{2})$, and nevertheless no true 4-partite entanglement is present.

If the subsystems happen to be of unequal dimension, then the respective subdimensions should be chosen not larger than the maximal allowed dimension such that all local density operators remain full rank. In a $2 \times 2 \times N$ system for example, a pure state can only have full support on the $2 \times 2 \times 4$ subspace, and therefore it makes no sense to calculate the normal form with $N>4$ : one can always first rotate the $N$-dimensional system into a 4-dimensional one by local unitary operations, and proceed by calculating the normal form for the $2 \times 2 \times 4$ system. More generally, if the dimension of the largest subsystem does not exceed the product of all the other ones, then generically the normal form will not be equal to zero, leading to non-trivial entanglement monotones. As an example, consider a $2 \times 2 \times 4$ system; there are more local SLOCC parameters than the number of degrees of freedom, so there will be only one entanglement monotone (as is the case in the $2 \times 2$ and $2 \times 2 \times 2$ case). The $2 \times 2 \times 4$ tangle is given by:

$$
\begin{aligned}
& \sqrt{4 / 3} \mid \sum \psi_{i_{1} j_{1} k_{1}} \psi_{i_{2} j_{2} k_{2}} \psi_{i_{3} j_{3} k_{3}} \psi_{i_{4} j_{4} k_{4}} . \\
& \left.\epsilon_{i_{1} i_{2}} \epsilon_{i_{3} i_{4}} \epsilon_{j_{1} j_{3}} \epsilon_{j_{2} j_{4}} \epsilon_{k_{1} k_{2} k_{3} k_{4}}\right|^{1 / 2}
\end{aligned}
$$

The factor $\sqrt{4 / 3}$ is included to ensure that the state in normal form

$$
(|000\rangle+|011\rangle+|102\rangle+|113\rangle) / 2
$$

has tangle given by 1 . Indeed, as will be shown in the following section, the maximal value of the tangle is always obtained for states in normal form, and this is the unique state (up to LU) having all its local density operators proportional to the identity. Note that this state is therefore the generalization of the $G H Z$ state to $2 \times 2 \times 4$ systems.

For completeness, let us also give a formula for the $2 \times 2 \times 3$ tangle:

$$
\begin{array}{r}
\sqrt[3]{\frac{27}{4}} \mid \sum \psi_{i_{1}, j_{1}, k_{1}} \psi_{i_{2}, j_{2}, k_{2}} \psi_{i_{3}, j_{3}, k_{3}} \psi_{i_{4}, j_{4}, k_{4}} \psi_{i_{5}, j_{5}, k_{5}} \psi_{i_{6}, j_{6}, k_{6}} . \\
\left.\epsilon_{i_{1} i_{4}} \epsilon_{i_{2} i_{5}} \epsilon_{i_{3} i_{6}} \epsilon_{j_{1} j_{4}} \epsilon_{j_{2} j_{5}} \epsilon_{j_{3} j_{6}} \epsilon_{k_{1} k_{2} k_{3}} \epsilon_{k_{4} k_{5} k_{6}}\right|^{1 / 3}
\end{array}
$$

The state maximizing this entanglement monotone (the number is bounded by 1) is the generalization of the $G H Z$ to the $2 \times 2 \times 3$ case:

$$
\frac{1}{\sqrt{3}}|000\rangle+\frac{1}{\sqrt{6}}|011\rangle+\frac{1}{\sqrt{6}}|101\rangle+\frac{1}{\sqrt{3}}|112\rangle .
$$

Let us finally give a non-trivial example of an entanglement monotone of the considered class in the case of three qutrits:

$$
\begin{gathered}
\sqrt{2} \mid \sum \psi_{i_{1} j_{1} k_{1}} \psi_{i_{2} j_{2} k_{2}} \psi_{i_{3} j_{3} k_{3}} \psi_{i_{4} j_{4} k_{4}} \psi_{i_{5} j_{5} k_{5}} \psi_{i_{6} j_{6} k_{6}} \\
\left.\epsilon_{i_{1} i_{2} i_{3}} \epsilon_{i_{4} i_{5} i_{6}} \epsilon_{j_{1} j_{2} j_{4}} \epsilon_{j_{3} j_{5} j_{6}} \epsilon_{k_{1} k_{5} k_{6}} \epsilon_{k_{2} k_{3} k_{4}}\right|^{1 / 3} .
\end{gathered}
$$

The other $\left(2 \cdot 3^{3}-1\right)-(3 \cdot 16)-1=4$ independent entanglement monotones can again be constructed by including more factors.

\section{Optimal Filtering}

A natural question now arises: characterize the optimal SLOCC operations to be performed on one copy of a multipartite system such that, with a non zero chance, a state with maximal possible multipartite entanglement is obtained. This question is of importance for experimentalists as in general they are not able to perform joint operations on multiple copies of the system. Therefore the procedure outlined here often represents the best entanglement distillation procedure that is practically achievable.

In the previous section a whole class of entanglement monotones that measures the amount of multipartite entanglement were introduced. The following theorem can easily be proved using the techniques of theorem [

Theorem 3 Consider a pure multipartite state, then the local filtering operations that maximize all entanglement monotones introduced in theorem 2 are represented by operators proportional to the determinant 1 SLOCC operations that transform the state into its normal form.

Proof: The proof of this theorem is surprisingly simple. Indeed, all the quantities introduced in theorem 2 are 
invariant under determinant 1 SLOCC operations if the states do not get normalized. The value of an entanglement monotone however only makes sense if defined on normalized states, and due to the linear homogeneity of the entanglement monotones, the following identity holds:

$$
M\left(\frac{\left(\otimes_{i} A_{i}\right) \rho\left(\otimes_{i} A_{i}\right)^{\dagger}}{\operatorname{Tr}\left(\left(\otimes_{i} A_{i}\right) \rho\left(\otimes_{i} A_{i}\right)^{\dagger}\right)}\right)=\frac{M(\rho)}{\operatorname{Tr}\left(\left(\otimes_{i} A_{i}\right) \rho\left(\otimes_{i} A_{i}\right)^{\dagger}\right)}
$$

The optimal filtering operators are then obtained by the $\left\{A_{i}\right\}$ minimizing

$$
\operatorname{Tr}\left(\left(\otimes_{i} A_{i}\right) \rho\left(\otimes_{i} A_{i}\right)^{\dagger}\right) .
$$

But this problem was solved in theorem 1 where it was proved that the $\left\{A_{i}\right\}$ bringing the state into its normal form minimize this trace.

It is therefore proved that the (reversible) procedure of washing out the local correlations maximizes the multipartite entanglement as measured by the generalization of the tangle. This is in complete accordance with the results of majorization [12, where it is shown that the notion of local disorder is intimately connected to the amount of entanglement present. Therefore we have supporting evidence to call pure states in normal form maximally entangled with relation to their SLOCC orbit.

\section{The mixed state case.}

The normal form derived in theorem 1 can readily be generalized to the case where the state is mixed, i.e. the case where the density operator is a convex sum of pure states. Indeed, nowhere in the proof of the theorem it was used that the state $\rho$ was pure; the same holds for the continuity for the normal form. We have therefore proven:

Theorem 4 Consider an $N_{1} \times N_{2} \times \cdots N_{m}$ mixed multipartite state. Then this state can be brought into a normal form by determinant 1 SLOCC operations, where the normal form has all local density operators proportional to the identity, and the normal form is unique up to local unitary operations. Moreover the trace of the normal form is the minimal one that can be obtained by determinant 1 SLOCC operations. If the SLOCC operations are chosen to be hermitian, then the normal form is continuous with respect to perturbations of the original state.

Note that if $\rho$ is full rank, its normal form will never converge to zero: the determinant of the density operator is constant under SLOCC operations.

It is also possible to adopt the results about entanglement monotones. First of all we extend the definition of an entanglement monotone $\mu_{p}$ that is defined on pure states and that is linearly homogeneous in $\rho$ by the convex roof formalism:

$$
\mu_{m}(\rho)=\min _{\sum_{i} p_{i}\left|\psi_{i}\right\rangle\left\langle\psi_{i}\right|=\rho} \sum_{i} p_{i} \mu_{p}\left(\left|\psi_{i}\right\rangle\right) .
$$

Here the optimization has to be done over all pure state decompositions of the state. The fact that the pure state entanglement monotone is linearly homogeneous in $\rho$ ensures that $\mu_{m}$ is, on average, not increasing under local operations, and therefore assures that $\mu_{m}$ is an entanglement monotone. Moreover, it is obvious that these entanglement monotones are again invariant under determinant 1 SLOCC operations. The results on optimal filtering for mixed states also readily apply, and therefore we arrive at the following very powerful result:

Theorem 5 The local filtering operations bringing a mixed state into its normal form are exactly the ones that maximize the entanglement monotones that remain invariant under determinant 1 SLOCC operations.

This result is remarkable, because there does typically not exist a way of actually calculating the value of an entanglement monotone defined on a mixed state: finding the optimal pure state decomposition of a state with relation to the convex roof formalism for a given EM is excessively difficult and has until now only been proven possible for the concurrence (i.e. the case of two qubits). So although we cannot calculate the entanglement monotone, we know how to maximize it! This particularly applies to mixed states of three qubits: we have proven how to maximize the 3-tangle, although we don't know how to calculate it.

Note that this optimal filtering procedure produces non-trivial results even in the case of two qubits: it proves that the concurrence and therefore the entanglement of formation of a mixed state of two qubits is maximized by the SLOCC operations bringing the state into its unique (Bell-diagonal) normal form.

\section{Conclusion}

In conclusion, we presented a constructive way of bringing a single copy of a quantum state into normal form under local filtering operations. This normal form is such that all local information is washed out (i.e. the local density operators are maximally mixed). We presented qualitative and quantitative arguments why the amount of entanglement of states in normal form cannot be enlarged by local operations, and introduced a whole class of entanglement measures that are a direct generalization of concurrence and 3-tangle to systems of arbitrary dimension. This sheds some new light on the difficulty encountered in classifying, understanding and unravelling the mysteries of multipartite quantum entanglement. 
We are very grateful to E. Briand, J-G. Luque and $\mathrm{J}-\mathrm{Y}$. Thibon for pointing out the theorems in algebraic geometry that prove the uniqueness of the normal form.

\section{APPENDIX: NORMAL FORMS UNDER LOCAL UNITARY OPERATIONS}

Consider a general multipartite state with $m$ parties defined on a $n_{1} \otimes n_{2} \cdots n_{M}$ dimensional Hilbert space:

$$
|\psi\rangle=\sum_{i_{1} \cdots i_{m}} \psi_{i_{1} \cdots i_{m}}\left|i_{1}\right\rangle\left|i_{2}\right\rangle \cdots\left|i_{m}\right\rangle .
$$

In this appendix, we try to solve the following natural question: is there a method to verify if two states $\left|\psi_{1}\right\rangle$ and $\left|\psi_{2}\right\rangle$ are equivalent up to local unitary transformations? In the bipartite case, this problem can readily be solved using the singular value decomposition, and we therefore ask for some kind of generalization of this diagonal normal form. Let us state the following theorem (see also Carteret et al. 17]), which is a weak generalization of the SVD:

Theorem 6 Given a general complex tensor $\psi_{i_{1} \cdots i_{m}}$ with dimensions $n_{1}=n_{2}=\cdots=n_{m}=n$, then there exist local unitaries $U_{i}$ such that all the following entries in the tensor $\psi^{\prime}=U_{1} \otimes \cdots \otimes U_{m} \psi_{i_{1} \cdots i_{m}}$ are set equal to zero:

$$
\begin{aligned}
\forall 1 \leq j \leq n, \forall k>j: & \psi_{j, j, \cdots, j, j, k}^{\prime}=0 \\
& \psi_{j, j, \cdots j, k, j}^{\prime}=0 \\
& \vdots \\
& \psi_{j, k, j, \cdots, j, j}^{\prime}=0 \\
& \psi_{k, j, \cdots, j, j}^{\prime}=0 .
\end{aligned}
$$

Moreover all entries $\psi_{n, n, \cdots, n, i, n, \cdots n}^{\prime}, i \leq n$ can be made real and positive. If the number of parties exceeds 2 , then the normal form is typically not unique up to permutations, but there exist a discrete number of different normal forms with the aforementioned property. The number of zeros however can generically not be increased by further local unitary operations.

Proof: unlike the proof in [17], this proof is constructive and can readily be translated into matlab code to calculate the normal form numerically. Consider first all entries with at least $m-1$ times 1 in its indices, and define the vectors $x_{i}^{1}=\psi_{i, 1,1, \cdots, 1}, x_{i}^{2}=\psi_{1, i, 1, \cdots, 1}, \ldots$, $x_{i}^{m}=\psi_{1,1, \cdots, 1, i}$. Define now a recursive algorithm that goes as follows: rotate $x^{1}$ to $\left\|x^{1}\right\|[1,0, \cdots 0]$ by a unitary transformation, apply the same transformtion on the full tensor, and define $x^{2}=\psi_{1, i, 1, \cdots, 1}$ with $\psi$ the transformed tensor. Now do the same thing with $x^{2}, \ldots x^{m}$ and then again with $x^{1}$, until the algorithm converges. This algorithm will certainly converge because at each step the
$(1,1, \cdots 1)$ entry of $\psi$ becomes larger and larger, unless all entries $(1,1, \cdots, 1, i, 1, \cdots 1)$ are equal to zero; moreover its value is bounded above because the unitary group is compact. Next exactly the same algorithm can be applied to the subtensor of $\psi$ defined as the one with all entries larger or equal to 2 (it is easy to check that the zeros obtained in the first step will remain zero by this kind of action). Next we can again do the same thing of another (smaller) subtensor, proving that indeed all zeros quoted in the theorem can be made.

It is straightforward to prove that the entries $\psi_{n, n, \cdots, n, i, n, \cdots n}^{\prime}, i \leq n$ can all be made real and positive by further diagonal unitary transformations.

Let us finally prove that no more zeros can be made by whatever unitaries (in the generic case). This follows from the fact that a unitary $n \times n$ matrix has $n^{2}$ continuous real degrees of freedom, but that only $n^{2}-n$ of them can be used to produce zeros as the other $n$ degrees of freedom can be imbedded in a diagonal unitary with just phases. Counting of the number of zeros produced indeed leads to

$$
\sum_{j=1}^{m} \sum_{k=1}^{m-1} \max (n-k, 0)=m \frac{n(n-1)}{2}
$$

which indeed corresponds to the $m\left(n^{2}-n\right)$ degrees of freedom as the zeros are "complex".

The non-uniqueness of the normal form obtained is surprising but can readily be verified by implementing the algorithm on a generic tensor; typically the algorithm converges to one out of a finite number of possible different normal forms.

As a first example, consider a system of three qubits. Unfolding the $2 \times 2 \times 2$ tensor in two $2 \times 2$ matrices, the following entries can always be made equal to zero:

$$
\left(\left(\begin{array}{ll}
x & 0 \\
0 & x
\end{array}\right)\left(\begin{array}{ll}
0 & x \\
x & x
\end{array}\right)\right)
$$

Here $x$ is used to denote a non-zero entry. In this case, it is easy to see that 4 of the remaining 5 entries can be made real by multiplying with appropriate diagonal local unitaries. This is equivalent to the normal form obtained by Acin et al. [18].

A more sophisticated example is the $3 \times 3 \times 3$ case, whose normal form looks like

$$
\left(\left(\begin{array}{lll}
x & 0 & 0 \\
0 & x & x \\
0 & x & x
\end{array}\right)\left(\begin{array}{lll}
0 & x & x \\
x & x & 0 \\
x & 0 & x
\end{array}\right)\left(\begin{array}{lll}
0 & x & x \\
x & 0 & x \\
x & x & x
\end{array}\right)\right)
$$

It is also straightforward to generalize the previous theorem (and constructive proof) to systems with different subdimensions (see Carteret et al. 17] for an existence proof); the algorithm of the previous proof can readily be extended to this case. Let us for example consider the 
normal form of the $N \times 2 \times 2$ case:

$$
\left(\left(\begin{array}{cc}
x & 0 \\
0 & x \\
0 & x \\
0 & x \\
0 & 0 \\
\vdots & \vdots \\
0 & 0
\end{array}\right)\left(\begin{array}{cc}
0 & x \\
x & x \\
x & 0 \\
0 & 0 \\
0 & 0 \\
\vdots & \vdots \\
0 & 0
\end{array}\right)\right)
$$

This case is of particular interest as it is describes a state of two qubits entangled with the rest of the world.

[1] C.H. Bennett, H.J. Bernstein, S. Popescu, and B. Schumacher. Concentrating partial entanglement by local operations. Phys. Rev. A, 53:2046-2052, 1996.

[2] N. Gisin. Hidden quantum nonlocality revealed by local filters. Phys. Lett. A, 210:151, 1996.

[3] M. Horodecki, P. Horodecki, and R. Horodecki. Inseparable two spin $1 / 2$ density matrices can be distilled to a singlet form. Phys. Rev. Lett., 78:574-577, 1997.

[4] N. Linden, S. Massar, and S. Popescu. Purifying noisy entanglement requires collective measurements. Phys. Rev. Lett., 81:3279, 1998.

[5] A. Kent. Entangled mixed states and local purification. Phys. Rev. Lett., 81:2839, 1998.

[6] A. Kent, N. Linden, and S. Massar. Optimal entanglement enhancement for mixed states. Phys. Rev. Lett., 83:2656, 1999.

[7] F. Verstraete, J. Dehaene, and B. De Moor. Local filtering operations on two qubits. Phys. Rev. A,
64:010101(R), 2001.

[8] W. Dür, G. Vidal, and J.I. Cirac. Three qubits can be entangled in two inequivalent ways. Phys. Rev. A, 62:062314, 2000.

[9] G. Vidal. Entanglement monotones. Jour. of Modern Optics, 47(2/3):355-376, 2000.

[10] V. Coffman, J. Kundu, and W.K. Wootters. Distributed entanglement. Phys. Rev. A, 61:052306, 2000.

[11] W.K. Wootters. Entanglement of formation of an arbitrary state of two qubits. Phys. Rev. Lett., 80:2245, 1998.

[12] M.A. Nielsen. Conditions for a class of entanglement transformations. Phys. Rev. Lett., 83:436-439, 1999.

[13] E. Briand, J-G. Luque and J-Y. Thibon, e-print quant-ph/0304026

[14] I.R. Shafarevich. Algebraic geometry IV Vol. 55 of Encycopedia of Mathematical Sciences; Springer-Verlag, Berlin, 1994.

[15] F. Verstraete, J. Dehaene, B. De Moor, and H. Verschelde. Four qubits can be entangled in nine different ways. Phys. Rev. A, 65:052112, 2002.

[16] A. Miyake. Topological classification of multipartite entangled states by the hyperdeterminant. 2002. quant-ph/0206111

[17] H.A. Carteret, A. Higuchi, and A. Sudbery. Multipartite generalization of the schmidt decomposition. J. Math. Phys., 41:7932, 2000.

[18] A. Acín, A. Andrianov, L. Costa, E. Jané, J. I. Latorre, and R. Tarrach. Schmidt decomposition and classification of three-quantum-bit states. Phys. Rev. Lett., 85:1560, 2000.

[19] Note that the numerical algorithm should not calculate $X$ from $\rho_{1}$ but instead from the singular value decomposition of the $N_{1} \times\left(\Pi_{i>1} N_{i}\right)$ matrix $\psi_{i_{1},\left(i_{2} \cdots i_{p}\right)}=U \Sigma V^{\dagger}$ : $X$ can be chosen as $X=\Sigma^{-1} U^{\dagger}$, and the numerical accuracy will be much higher. 